\title{
ERRATUM
}

Unfortunately, the following paper was missing from the Proceedings of the International Conference on the Application of the Mössbauer Effect (Leuven, 1985); Hyp. Int. 29(1986).

\section{LIMITS OF APPLICABILITY OF THE CLASSICAL FIELD CONCEPT IN MÖSSBAUER SPECTROSCOPY}

\author{
J. JAVANAINEN ${ }^{1,2}$, P. HELISTÖ ${ }^{3}$, E. IKONEN $^{3}$ and T. KATILA ${ }^{3}$ \\ 'Max-Planck-Institut für Quantenoptik, D-8046 Garching, West Germany \\ ${ }^{2}$ University of Helsinki, Research Institute for Theoretical Physics SF-00170 Helsinki 17, Finland \\ ${ }^{3}$ Department of Technical Physics, Helsinki University of Technology SF-02150 Espoo 15, Finland
}

We show that the classical model widely employed for the field radiated by an excited $M \ddot{s}$ sbauer nucleus predicts an enhanced rate of coincidences for two detectors. This contradicts our experiment. We discuss the limits of applicability of the classical field concept for various experimental conditions.

\section{INTRODUCTION}

It is standard practice in Mössbauer spectroscopy to assume that a source nucleus excited at time $t_{0}$ radiates an exponentially decaying classical field whose positive frequency part is

$E_{S}\left(t ; t_{0}\right) \propto \theta\left(t-t_{0}\right) \exp \left\{-\left(i \omega+\frac{\Gamma}{2}\right)\left(t-t_{0}\right)-i \frac{2 \pi \times(t)}{\lambda}-i \phi\right\}$

Here $\omega$ is the transition frequency and $\lambda$ the wavelength, $1 / \Gamma$ is the lifetime of the excited level, $x(t)$ accounts for the assumedly one-dimensional motion of the source, and $\phi$ is a phase varying randomly from one nucleus to another that is added in order to prevent unphysical interference of the fields from different nuclei. This model has successfully been applied to analyze results obtained e.g. in coincidence experiments $/ 1$, and in recent quantum beat and transient measurements $/ 2-6 /$. Also the conventional transmission integral can be derived from it. Nevertheless, Eq. (1) amounts to describing spontaneous emission in terms of a classical field, a procedure which is certainly not sound.

In this contribution we address the validity of the classical field model. We first show that it is not universally valid: When the field of the source is monitored with two detectors, the model predicts an enhanced coincidence rate which contradicts our experiment. Second, we approach the question why the classical model still seems to give correct results as long as intensities, but not intensity correlations, are ultimately recorded in an experiment. 


\section{2.' FAILURE OF THE CLASSICAL FIELD MODEL}

Where the classical field model has been used, the counting rate of a detector, i.e. the intensity of the field after traversing the absorber, has typically been observed. However, the intensity does not completely characterize the electromagnetic field. In search for a counterexample to $\mathrm{Eq} .(1)$ we therefore study the simplest possible arrangement which goes beyond the measurement of the intensity: a source and two detectors to examine coincidences of photon counts. According to the theory of photoelectric detection $/ 7 /$, the joint counting rate of the two detectors at times $t_{1}$ and $t_{2}$ is proportional to the intensity correlation function

$$
C(2)\left(t_{1}, t_{2}\right)=\left\langle\left|E_{1}\left(t_{1}\right)\right|^{2}\left|E_{2}\left(t_{2}\right)\right|^{2}\right\rangle
$$

Here $E_{1}\left(E_{2}\right)$ is the sum of the positive frequency parts of the fields from the individual source nuclei at detector 1 (2). The brackets stand for averaging over the creation times and

\section{APPLICABILITY OF THE CLASSICAL FIELD MODEL}

The classical field model gives an incorrect picture of our coincidence measurement, yet it is supported by a large amount of data in Mössbauer spectroscopy. In order to study when it is legitimate to use the classical theory we compare it with QED.

Let us first consider a single emitter nucleus excited at a fixed time $t_{0}$ and an absorber containing any number of nuclei, assuming that the source does not move with respect to the absorber. We start with the classical case. In order to determine the field at the detector the propagation of the field through the absorber has to be examined. That is, the coupled Maxwell equations for the field and the Bloch equations for the medium must be solved $/ 9 /$. Since in Mössbauer spectroscopy the field strengths are too low to create a considerable population in the excited levels of the absorber nuclei, saturation in the sense of laser spectroscopy does not occur. Hence the Maxwell-Bloch equations are linear, and the field at the detector is a linear mapping of the source field:

$E_{D}\left(t ; t_{0}\right)=\int_{-\infty}^{t} d t^{\prime} K_{C l}\left(t-t^{\prime}\right) E_{S}\left(t^{\prime} ; t_{0}\right)$,

where $K_{c l}(t)$ is the response function of the absorber. The intensity at the detector is thus

$I_{D, C l}\left(t_{;} ; t_{0}\right)=\left|E_{D}\left(t ; t_{0}\right)\right|^{2}=\int_{-\infty}^{t} d t^{\prime} \int_{-\infty}^{t} d t^{\prime \prime} K_{C l}^{*}\left(t-t^{\prime}\right) K_{C l}\left(t-t^{\prime \prime}\right) E_{S}^{*} S^{\prime}\left(t^{\prime} ; t_{0}\right) E_{S}\left(t^{\prime \prime} ; t_{0}\right)$.

The QED version of the theory is phrased in terms of the Heisenberg picture operators for the field and the nuclei. The source field is proportional to the positive frequency part of the dipole (or some higher multipole) operator of the source nucleus $/ 10 /, \hat{E}_{S}\left(t ; t_{0}\right) \propto d\left(t ; t_{0}\right)$. The theory of the interaction of the field with the absorber nuclei can be formulated in $Q E D$ using the quantum versions of the Maxwell-Bloch equations $/ 11 /$. In the absence of saturation they are linear; they are actually the same equations as in the classical theory, except that they involve noncommuting operators. By virtue of linearity, the quantum field at the detector should be expressible in the form of Eq.(4) with some propagator $K_{q m}(t)$. According to the theory of photodetection the intensity (the probability of a photon count as a function of time) is then 
$I_{D, q m}\left(t ; t_{0}\right)=\left\langle\hat{E}^{\dagger} D\left(t ; t_{0}\right) \hat{E}_{D}\left(t ; t_{0}\right)\right\rangle \propto \int_{-\infty}^{t} d t^{\prime} \int_{-\infty}^{t} d t^{\prime \prime} K_{q m}^{*}\left(t-t^{\prime}\right) K_{q m}\left(t-t^{\prime \prime}\right)\left\langle d^{\dagger}\left(t^{\prime} ; t_{0}\right) d\left(t^{\prime \prime} ; t_{0}\right)\right\rangle .(6)$

This is a functional of the two-time Heisenberg picture expectation value of the dipole operator, i.e. of the dipole correlation function.

A comparison of Eqs. (5) and (6) shows that the classical and QED results for the intensity are the same, except for trivial factors, if

$K_{c l}(t)=K_{q m}(t)$

$E_{S}^{*}\left(t ; t_{0}\right) E_{S}\left(t^{\prime} ; t_{0}\right)=\left\langle\partial t\left(t ; t_{0}\right) \partial\left(t^{\prime} ; t_{0}\right)\right\rangle$.

But because the Maxwell-Bloch equations have the same form in classical and QED theories, it seems plausible that the propagators are the same and that condition $(7 \mathrm{a})$ is valid. On the other hand, the left-hand side of Eq.(7b) is obtained from Eq.(1) and the dipole correlation random phases of the emitting nuclei, and over the positions of the source nuclei and detector elements. We emphasize that the fields E in Eq.(2) a re classical; to get "photon counts" it is sufficient to quantize the detectors $/ 8 /$.

Using Eqs. (1) and (2), the correlation function becomes

$G^{(2)}\left(t_{1}, t_{2}\right)=\left.I_{1}\right|_{2}\left\{1+\frac{\Gamma}{2 \eta} \exp \left(-\Gamma\left|t_{1}-t_{2}\right|\right)\right\}$,

where $\left.\right|_{1,2}=\left\langle\left|E_{1,2}(t)\right|^{2}\right\rangle$ are the intensities at the detectors and $n$ is the activity of the source. At zero time difference $\left(t_{1}-t_{2}=0\right)$ the two detectors should thus record $\Gamma / 2 n$ times more coincidence counts than for a large time separation $\left(\left|t_{1}-t_{2}\right|+\infty\right)$. Moreover, the probability of excess coincidences should fall of exponentially as a function of $\left|t_{1}-t_{2}\right|$ with the time constant $\Gamma^{-1}$.

To test this prediction we have carried out a delayed coincidence experiment on the field emanating from a ${ }^{151} \mathrm{Sm}$ source, with $1 / \Gamma=14 \mathrm{~ns}$ and the gamma activity of the source $\eta=1.1 \cdot 10^{6} \mathrm{~s}^{-1}$. The time resolution of our apparatus was $\approx 3.5 \mathrm{~ns}$, and the overall signal-

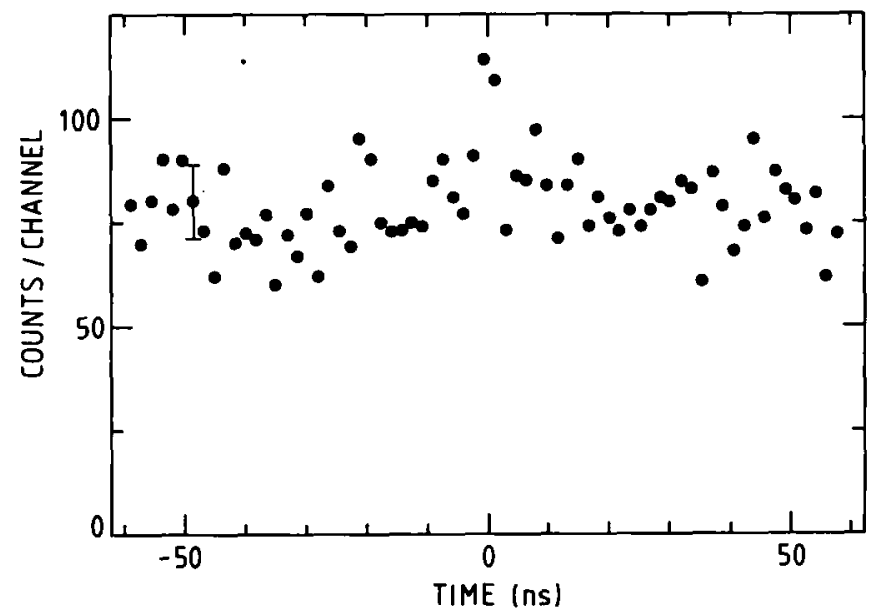

Fig.1. The joint counting rate at two detectors as a function of the time separation for a ${ }^{151} \mathrm{Sm}$ source. 
to-background ratio was $\approx 0.4$. The result of a one-week run is shown in Fig. 1 .

At first sight the two channels around zero time difference rising above the constant level of counts in Fig. 1 might be confused with coincidences predicted by Eq.(3). However, this peak was strongly enhanced by removing the $\mathrm{Pb}$ shield mounted between the detectors. Thus we ascribe the peak to scattering of radiation from one detector to the other. The excess coincidences of Eq. (3), which should have the FWHM of $\simeq 20$ ns and height of about 1000 counts/channel, are clearly absent in Fig.1. Even if the more conservative assumption is adopted that only the recoilless fraction of the intensity $f=0.44$ contributes to the bunching and hence the factor in front of the exponential in Eq. (3) is multiplied by $f^{2}$, about 250 counts/channel in the middle of Fig. 1 are still expected. The classical field model of Eq.(1) fails in explaining the outcome of our experiment.

We have not given details of the derivation of Eq.(3), but to isolate the reason for the discrepancy with the experiment, it suffices to note that the extra coincidences derive from events where emission of one nucleus gives rise to a count on both detectors. This contradicts the notion that the photons emitted by the nuclei are indivisible entities. In fact, a full quantum-electrodynamical (QED) analysis of our scheme gives simply $G(2)=I_{1} I_{2}$, which agrees with the experiment.

function is known in quantum optics $/ 11 /$. It is easy to verify that condition (7b) is satisfied.

The restriction to a single source nucleus is superfluous since the intensities from the emitters add in both classical and quantum theories. Moreover, when the excitation time $t_{0}$ is not observed, an integral over it has to be carried out, but this is the same in both theories. The relative motion of the source and the absorber only introduces phase factors which are again the same in classical and QED theories.

Summarizing, if the quantum propagator $K_{\text {qm }}$ exists and is the same as the classical propagator $\mathrm{K}_{\mathrm{cl}}$, the nontrivial result of $\mathrm{Eq} .(\mathrm{lb})$ on the dipole correlation function guarantees that the classical approach and QED predict the same intensity. Within a different formalism Harris $/ 12 /$ has essentially shown that under the conditions of the quantum beat and transient experiments carried out so far, the assertion about the propagators is valid. We suggest that the equality of the propagators reflects an isomorphism of classical and quantum theories, and by utilizing this fact his assumptions can be relaxed. The major difficulty of such an endeavor is that QED is a truly microscopic theory, hence a rigorous comparison is only possible if both the classical and the quantum theories are developed on the level of the individual nuclei.

\section{CONCLUDING REMARKS}

Our measurement of intensity correlations shows that the classical model does not describe correctly the field from a Mössbauer source nucleus. Nevertheless, it seems to be applicable at least to all published quantum beat and transient experiments. We anticipate that, by developing our novel approach to comparison of classical and quantum theories, we will be able to widen the scope of the classical field model considerably.

References

$/ 1 /$ F.J. Lynch, R.E. Holland and M. Hamermesh, Phys. Rev. 120 (1960) 513.

/2/ G.J. Perlow, Phys. Rev. Lett. 40 (1978) 896.

13/ J.E. Monahan and G.J. Perlow, Phys. Rev. A 20 (1979) 1499.

/4/ P. Helistö, E. Ikonen, T. Katila and K. Riski, Phys. Rev. Lett. 49 (1982) 1209.

15/ P. Helistö, E. Ikonen, T. Katila, W. Potzel and K. Riski, Phys. Rev. B 30 (1984) 2345.

16/ E. H. du Marchie van Voorthuysen, G. L. Zhang and H. de Waard, Phys. Rev. A 30 (1984) 2356.

17/ R. J. Glauber in 'Quantum Optics and Electronics", eds. C. De Witt, A. Blandin and C. Cohen-Tannoudji (Cordon and Breach, New York, 1965).

18/ L. Mandel in "Progress in Optics", Vol. XIII, ed E. Wolf (North-Holland, Amsterdam, 1976).

19/ L. Allen and 1. H. Eberly, 'Optical Resonance and Two-Level Atoms' (Wiley, New York, 1975).

/10/ B. R. Mollow, Phys. Rev. 188 (1969) 1969.

111 J. R. Ackerhalt and J. H. Eberly, Phys، Rev. D 10 (1974) 3350.

/12/ S. M. Harris, Phys. Rev. 124 (1961) 1178. 